\title{
Fabrication, characterization and gas sensing properties of gold nanoparticle and calixarene multilayers
}

\author{
INCI ÇAPAN ${ }^{1, *} \mathbb{D}$, ASEEL K HASSAN ${ }^{2}$ and RAJAA R ABBAS ${ }^{3}$ \\ ${ }^{1}$ Faculty of Art and Sciences, Department of Physics, Balıkesir University, Cagis Campus, 10145 Balikesir, Turkey \\ ${ }^{2}$ Faculty of Arts, Computing, Engineering and Sciences, Sheffield Hallam University, Sheffield S1 2NU, \\ United Kingdom \\ ${ }^{3}$ Department of Physiology and Medical Physics, College of Medicine, Kufa University, Kufa, Iraq
}

MS received 8 September 2015; accepted 23 May 2016

\begin{abstract}
Calixarenes are a group of materials that are widely used for gas sensing studies because of their simple synthesis, conformational flexibility, binding group tunability, variability in their cavity sizes and improved selectivity to different gas molecules. In recent years it has been shown that incorporation of gold nanoparticles (AuNPs) into organic layers further enhances their gas sensing performance. The present study reports on the fabrication of thin films of calixarene and AuNPs using Langmuir-Schaefer (LS) methods. The gas sensing properties of the produced films are investigated on exposure to saturated vapours of volatile organic compounds (VOCs) using surface plasmon resonance as an optical detection technique. Multilayers comprising films of AuNPs and calixarene have been investigated to evaluate the effect of AuNPs on the films sensing performances. It has been demonstrated that the hybrid layers exhibited improved sensing performance in terms of the degree of their response.
\end{abstract}

Keywords. Sensors; surface plasmon resonance; gold nanoparticle; thin film.

\section{Introduction}

In recent years, AuNPs, possessing high chemical stability, facile synthesis, surface functionalization, biocompatibility and tunable optical and electronic properties, have led to many promising applications in sensing area $[1,2]$. To act as a selective probe, the nanoparticles are subjected to functionalization with a receptor molecule. The surfaces of graphene thin films integrated with AuNPs have been presented as an attractive approach for gas detection applications [3]. It has also been demonstrated that the AuNPs/reduced graphene oxide hybrid thin film shows a promising micro-gravimetric sensing towards ammonia gas [4]. Enhanced sensing performance of $\mathrm{TiO}_{2} /$ gold nanocomposite thin films has been demonstrated using optical gas sensing techniques [5]. The role of gold catalyst on the sensing behaviour of $\mathrm{ZnO}$ nanorods and nanoplates has also been demonstrated through the functionalization of $\mathrm{ZnO}$ nanorods with noble metals; this hybrid was shown to solve the problem of selectivity between reducing and oxidizing gases [6,7]. Goldfluoropolymer $(\mathrm{CFx}(\mathrm{Au}))$ nanocomposites having different gold contents also reveal a higher sensitivity towards organic compounds for the nanocomposites with higher content of gold [8]. The detection of metal ions has also been achieved using an AuNP-based hybrid nanosystem in aqueous solution for the determination of $\mathrm{Hg}$ (II) [9].

\footnotetext{
*Author for correspondence (inci.capan@gmail.com,
} ibasaran@balikesir.edu.tr)
The cavity of the calixarene provides a site for the binding of organic guest molecular species and the selectivity of calixarenes to particular analytes can be controlled somehow by altering the size of the cavity [10] as well as the peripheral substituted groups [11]. The calixarene molecules are excellent thin-film-forming materials to be used as host molecules to detect both organic $[12,13]$ and inorganic gases $[14,15]$. The detection of a binary mixture of butylamine isomers has been achieved by self-assembled calixarene bilayer films on piezoelectric quartz crystals [16]. Moreover the detection of organic amine vapours was also demonstrated by the optical chromogenic effect, which was found to be determined by the basicity and the steric hindrance of the amine [17]. Calixarene layers are also known to be sensitive and selective to inorganic vapours such as carbon dioxide and carbon monoxide [18].

Due to their excellent sensing capabilities calixarene molecules are selected as probes to be functionalized by AuNPs. The decoration of organic microstructures of resorcinarene molecules, both inside and outside, with metal nanoparticles has been described in detail $[19,20]$. The AuNP-functionalized calixarene molecules were found to be promising materials for detection of metal ions [21,22].

In this work AuNPs thin films, calixarene bilayer films and AuNPs-calixarene hybrid films have been fabricated via Langmuir-Schaefer (LS) thin-film fabrication techniques. UV-visible spectroscopy and surface plasmon resonance (SPR) technique have been employed for the characterization of the prepared thin films. SPR is known as a highly sensitive 
method for the determination of bio-analytes, where commercial SPR systems (e.g., Reichert4SPR 4 Channel SPR system) are in use, which provide real-time determination of interactions and kinetic studies. The SPR sensor has various advantages such as high sensitivity, good selectivity, low cost [23], do not need electrical contacts and do not suffer from electromagnetic noise [24]. The experimentally measured SPR curves were fitted using the Winspall software to evaluate the films' thickness as well as their optical parameters ( $n$ and $k$ ). To the best of our knowledge the thickness and optical parameters of hybrid thin films of calixarene molecules deposited onto AuNPs monolayer are reported for the first time. The gas sensing properties of the hybrid films have been investigated by measuring the shift of the maxima of the UV-visible spectra as well as the SPR shift of the thin films under exposure to saturated vapour concentrations of the gas molecules.

\section{Experimental}

\subsection{Materials}

Commercial dodecanethiol $\left(\mathrm{CH}_{3}\left(\mathrm{CH}_{2}\right)_{11} \mathrm{SH}\right)$-encapsulated gold nanoparticles (AuNPs) with $2 \mathrm{~nm}$ particle size (purchased from PlasmaChem GmbH, Germany) [25] and calix[4]arene-17C were used in this work. The chemical structures of the AuNPs and calixarene molecules can be found elsewhere [26,27]. Hexamethyldisilazane (HMDS) was purchased from Sigma-Aldrich, while chloroform $\left(\mathrm{CHCl}_{3}\right)$ and isopropanol (IPA) were obtained from Fisher Scientific. The analyte gas molecules selected in the present work were benzene, toluene, chloroform and dichloromethane (DCM) because of their toxicity and harmful effects on human health; all of them were purchased from Sigma-Aldrich.

\subsection{LS film fabrication and characterization}

Gold-thiolated solution was prepared using chloroform $\left(\mathrm{CHCl}_{3}\right)$ as solvent with the concentration of $1 \mathrm{mg} \mathrm{ml}^{-1}$. The solution was used for the deposition of LS monolayer films at the water-air interface in a computer-controlled trough (NIMA-Type: 611). The Wilhemly plate was used to measure the surface pressure of the nanoparticles' monolayer. After evaporation of solvent for about 5-10 min, the floating film resulting from spreading $500 \mu \mathrm{l}$ of solution on the water surface was compressed at a rate of $20 \mathrm{~cm}^{2} \mathrm{~min}^{-1}$ and the corresponding isotherms were acquired. AuNPs films were deposited onto glass substrates by using LS deposition at a surface pressure of $18 \mathrm{mN} \mathrm{m}^{-1}$. The substrates were cleaned prior to deposition by chloroform and then IPA and distilled water, dried with $\mathrm{N}_{2}$ flux, and finally treated with HMDS to be hydrophobic. All these procedures were carried out in a Class 10,000 clean room at room temperature. The calixarene thin film and the hybrid thin film were fabricated using the same procedure for multilayer fabrication.

\subsection{Gas sensing experiments}

The SPR technique measures the light intensity as a function of the angle of incidence between two media, which consists of a metal and a dielectric. The dielectric properties of the sensing layer (mainly index of refraction and extinction coefficient) as well as its thickness can be significantly influenced by the properties of the medium interfacing the sensing layer that is deposited onto the surface plasmon supporting layer $(\mathrm{Au})$. To investigate the gas sensing properties of the sensing layer a resonance condition is established at a certain angle of incidence of a p-polarized light beam (typically from a He-Ne laser source), which is sensitive to the properties of this layer and its surrounding ambient. The change in light intensity as a function of time due to gas exposure is monitored around the resonance angle position by fixing the angle of incidence. A Biosuplar 6 Model spectrometer with a low-power laser diode $(630-670 \mathrm{~nm})$ light source was employed to monitor the SPR curves with an angular resolution of about $0.003^{\circ}$. A glass prism $(n=1.62)$ was mounted within a holder and a compatible transparent plastic flow cell with an inlet and outlet connected to silicone tubes was used for organic vapour injection. A schematic diagram of the SPR system can be found elsewhere [28].

\section{Results and discussion}

\subsection{Films' fabrication and characterization}

As a first step for the fabrication of LS thin films of the AuNPs and the calixarene molecules, pressure-area (П-A) isotherm graphs have been recorded to determine the appropriate deposition surface pressure values of the monolayers. The $\Pi$-A isotherm graphs of the AuNPs molecules can be found from our previous publication [26]. All monolayers with different volumes of solution spread onto the water surface form stable monolayers at the air-water interface. Gas, liquid and solid phases with the collapse pressure of the monolayer are clearly seen in the isotherms during the compression of the monolayer.

Figure 1 shows the UV-visible spectra of thin films with 1 layer of AuNPs, 2 layers of calixarene as well as hybrid films that consist of 1 layer of AuNPs and 2 layers of calixarene molecules on top. The UV-visible spectra of the AuNPs monolayer show a broad absorption band with a maximum centred around $584 \mathrm{~nm}$ at room temperature. Previous studies have shown that the maximum absorbance of 4-methylbenzenethiol-encapsulated $3 \mathrm{~nm}$ AuNPs in chloroform solution was around $515 \mathrm{~nm}$. AuNPs films transferred onto glass substrate however have exhibited a red-shift to about $575 \mathrm{~nm}$ at room temperature [29,30]. This redshift is believed to be the result of aggregation of AuNPs on the substrate surface. The UV-visible spectra of the calixarene bilayer show a typical absorbance maximum at $353 \mathrm{~nm}$, which is consistent with the literature [13]. The UVvisible spectra of the hybrid film reveal a broad maximum absorbance value at $540 \mathrm{~nm}$, which has also been reported 


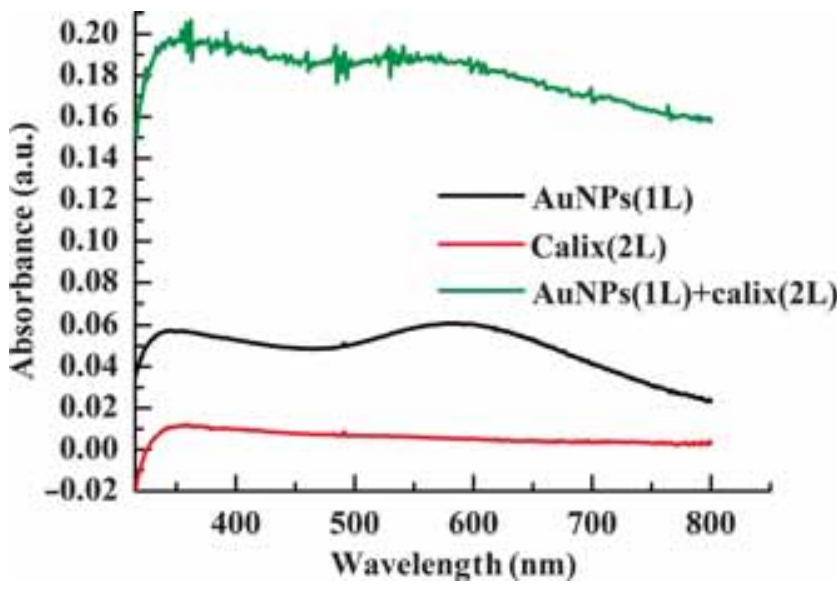

Figure 1. UV-visible spectra of thin films with 1 layer of AuNPs, 2 layers of calixarene and the hybrid film.

for AuNPs $/ \mathrm{TiO}_{2}$ nanocomposite thin films [5]. The successful preparation of the AuNPs-calixarene hybrid films is confirmed by the UV-visible spectra as shown in figure 1 . The characteristic absorbance maxima of AuNPs layer are confirmed both in AuNPs monolayer thin film and in the AuNPs-calixarene hybrid film, which implies the calixarene layers transfer onto the AuNPs monolayer.

Similar films have also been deposited onto $40 \mathrm{~nm}$ thick gold-coated substrates for the investigation of their gas sensing properties using SPR method. The SPR curve measures the reflected light intensity as a function of the angle of incidence. Figure 2 shows SPR curves recorded over the angle range $\theta=41-52^{\circ}$ for the different layer structures studied in this work. The minima in the reflected light intensity occur at the resonance angle $\theta_{\mathrm{SPR}}$, which is expressed as

$$
\theta_{\mathrm{SPR}}=\sin ^{-1}\left(\frac{\varepsilon_{\mathrm{M}}}{\varepsilon_{\mathrm{P}}\left(\varepsilon_{\mathrm{M}}+1\right)}\right)^{1 / 2},
$$

where $\varepsilon_{\mathrm{M}}$ is the dielectric constant of the metal film sandwiched between a glass prism of dielectric constant $\varepsilon_{\mathrm{P}}$ [31] and air. A shift in the resonance angle $\theta_{\mathrm{SPR}}$ occurs when a thin film with a thickness $d$ and dielectric constant $\varepsilon$ is deposited onto the metal film. This shift $\Delta(\theta)$ is given by [32]

$$
\Delta \theta=\frac{(2 \pi / \lambda)\left(\left|\varepsilon_{\mathrm{M}}\right|\right)^{3 / 2} d}{\sqrt{\varepsilon_{\mathrm{P}}} \cos \theta\left(\left|\varepsilon_{\mathrm{M}}-1\right|\right)^{2} \varepsilon}(\varepsilon-1),
$$

where $\left|\varepsilon_{M}\right|$ is the modulus of the real part of the dielectric constant of the metal film and $\varepsilon$ is the dielectric constant of the film. It is clear that the shift in the resonance angle is dependent on the thickness and the dielectric constant of the film deposited onto the Au layer.

The transfer quality as well as thicknesses and the optical constants of the thin films can be assessed using their SPR curves. The shift in $\theta_{\text {SPR }}$ with respect to the bare gold indicates the successful transfer of the AuNPs as well as the calixarene films onto the Au-coated slides. The maximum shift of $3.25^{\circ}$ in the resonance angle was obtained for the

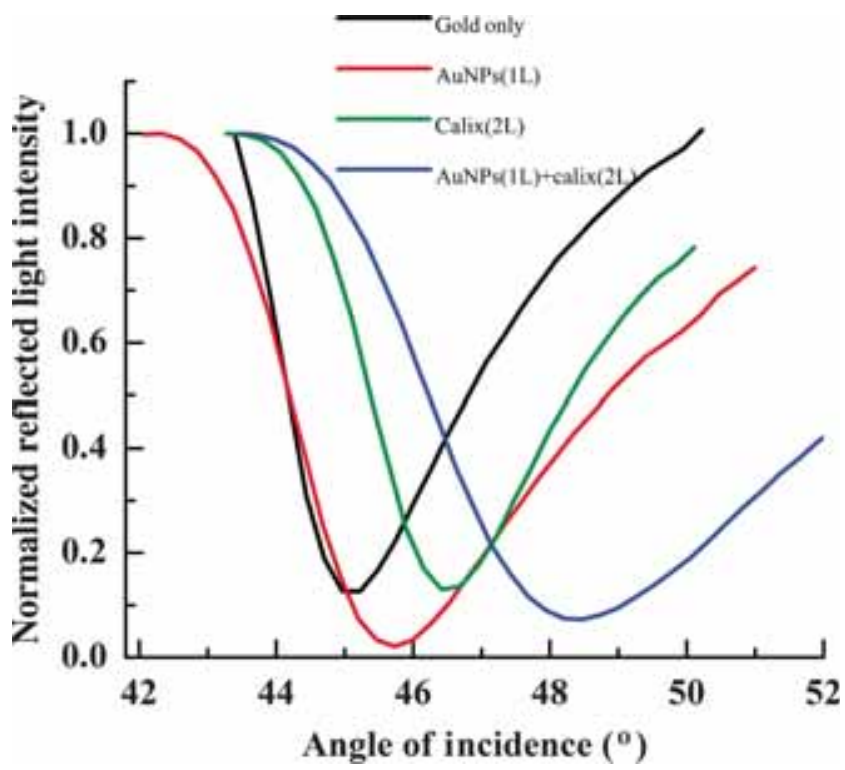

Figure 2. SPR curves of 1 layer of AuNPs, 2 layers of calixarene and the hybrid film with uncoated gold substrate.

AuNPs-calixarene hybrid film, whereas a smaller shift of about $0.6^{\circ}$ was observed for the AuNPs monolayer.

The experimental SPR data were fitted using the Winspall software (written by Wolfgang Knoll, developed at the MaxPlank-Institute for Polymer Research, Germany) in order to evaluate the different films' parameters, which include their thickness and refractive index, both real and imaginary parts. The SPR curve for the bare gold film is used to determine the gold layer thickness and optical constants; they were then used during the fitting of the other films' SPR curves to deduce their parameters. The gold layer thickness was found to be $42 \pm 0.04 \mathrm{~nm}$, while $n$ and $k$ were 0.18 and 3.35, respectively, which is consistent with the literature [33]. The AuNPs monolayer thickness of $2.36 \mathrm{~nm}$ and optical parameters $n=2.18$ and $k=0.35$ obtained at $\lambda=632 \mathrm{~nm}$ are in good agreement with the previous reports [30]. The calixarene bilayer thickness was found to be $5.1 \mathrm{~nm}$. The previous studies on the thickness of the LB thin films of calixarene molecules report a maximum thickness value of $3.5 \mathrm{~nm}$ per bilayer [34]. Our fitting results give a higher value, which may be caused by different orientation of the molecules on the substrate. Refractive index ( $n$ ) values between 1.64 and 1.82 of calixarene films were reported [13] and our SPR data fitting for similar films give a refractive index value of 1.7, which falls in the range of reported data. The AuNPscalixarene hybrid layer properties have also been evaluated using the Winspall curve fitting, which yields a thickness value of $6.22 \mathrm{~nm}$, and optical constants $n=2.61$ and $k=0$.

\subsection{Gas sensing experiments}

UV-visible spectroscopy as well as SPR method have been employed to investigate the response of the different films' structures on exposure to the studied analytes. 
The UV-visible spectra of the unexposed hybrid film are presented with the UV-visible spectra of the same thin film under exposure to different vapours in figure 3 . For the unexposed hybrid film the maximum absorbance has been observed to occur in two different positions: at 362 and $541 \mathrm{~nm}$. It was also shown that when the hybrid film is exposed to different volatile organic compounds (VOCs) these positions might change. The position of the first maxima at $362 \mathrm{~nm}$ was shifted by $3 \mathrm{~nm}$ on exposure to benzene and toluene, while no changes were observed during exposure to the other analytes. The second absorption maximum at $541 \mathrm{~nm}$ exhibits a red-shift of 16, 23, 26 and $46 \mathrm{~nm}$ as a result of exposure to DCM, chloroform, toluene and benzene gases, respectively. A similar behaviour has been reported for gold nanoisland thin films in the presence of ozone

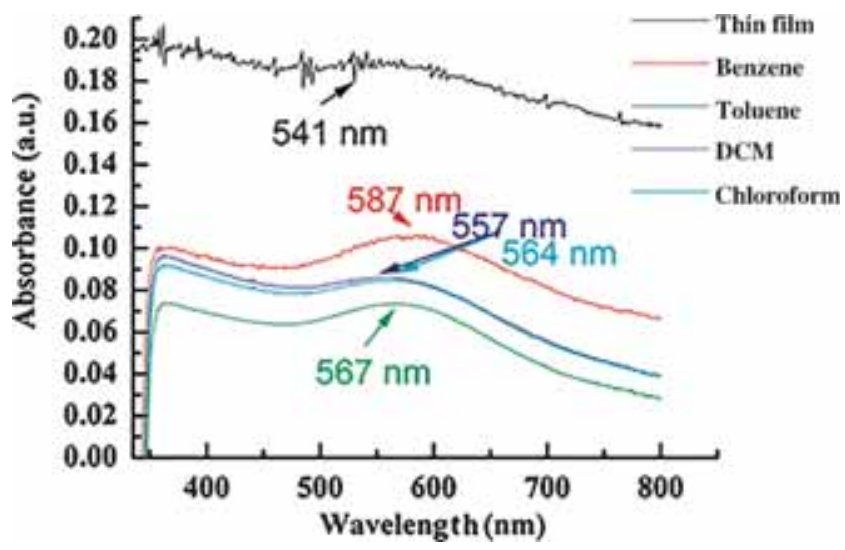

Figure 3. The UV-visible spectra of the unexposed hybrid film and the thin film under exposure to saturated benzene, toluene, DCM and chloroform vapours. gas [35]. The maximum change was observed for benzene exposure, while the minimum change was associated with DCM exposure.

The kinetic measurements for the AuNPs monolayer, calixarene bilayers and their hybrid film obtained using SPR method under exposure to saturated vapour of chloroform are presented in figure 4 . The organic vapours are injected into the gas cell and left for $2 \mathrm{~min}$ to interact with the sensing layer. The interaction is based on the physical adsorption of the analyte molecules to the sensing layer surface and it is known to be reversible as has been published by many researchers [36-39]. The inset shows the kinetic response of the hybrid film obtained during two consecutive exposures of saturated vapour of chloroform, which shows clear reproduciblity of sensor response. Similar results were obtained for exposures to other analyte vapours (results are not included here). The normalized maximum response for all tests of gas exposures has been listed in table 1. It is shown that the hybrid film reveals the highest responses among the three different films under investigation, except for the benzene gas exposure. This can be ascribed to the enhanced interaction properties of the hybrid film and thus its improved sensitivity to organic vapour exposures. The highest response was observed for chloroform vapour, while the lowest was due to benzene vapour exposure. The response and recovery times were between 2-3 and 15-20 s, respectively.

Using two different optical detection techniques has revealed different types of responses towards the same gases. Investigation performed using the UV-visible spectroscopy has shown the highest response towards benzene, which is known with its non-polar character, while the lowest response was obtained for DCM, which is a polar solvent. Previous studies on similar thiolated AuNP thin films have

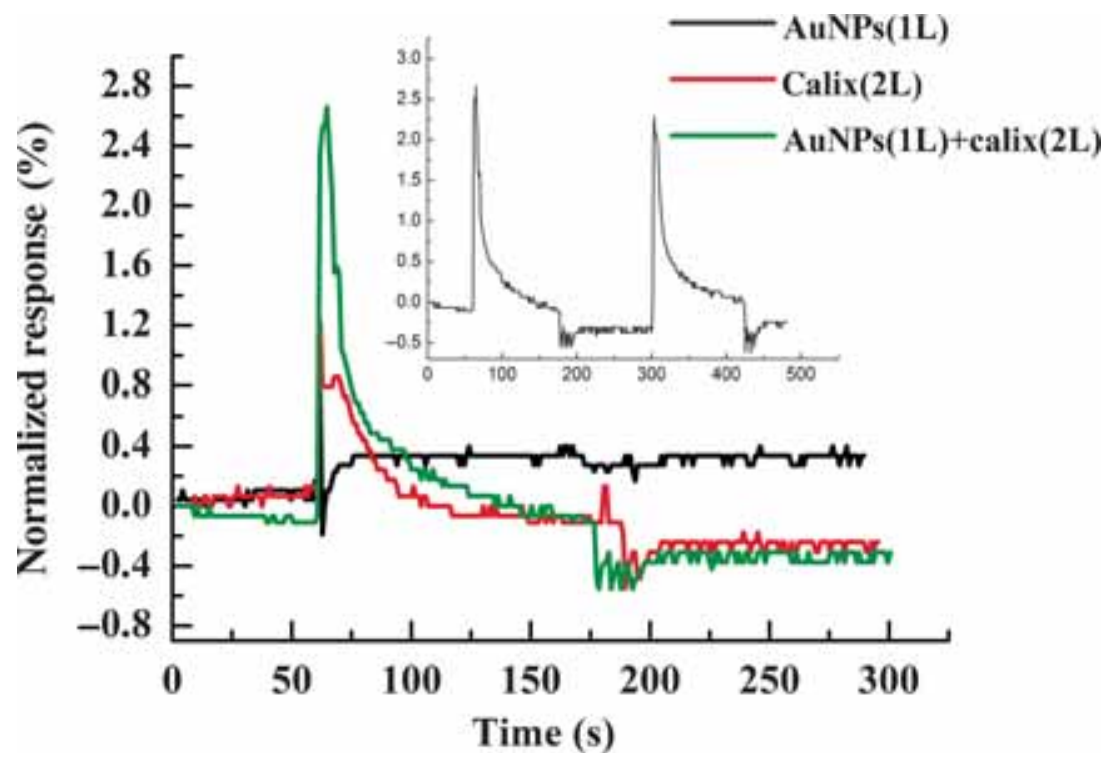

Figure 4. Kinetic gas response for the AuNPs monolayer, calix bilayers and their hybrid film obtained using SPR method under exposure to saturated vapour of chloroform. Inset: the kinetic response of the hybrid film obtained during two consecutive exposures of saturated vapour of chloroform. 
Table 1. The normalized maximum response for all tests of gas exposures.

\begin{tabular}{lcccc}
\hline & \multicolumn{4}{c}{ Normalized response (\%) } \\
\cline { 2 - 5 } Thin films & Benzene & Toluene & Chloroform & DCM \\
\hline AuNPs (1L) & 0.27 & 0.81 & 0.62 & No signal \\
Calix (2L) & 1.93 & 0.81 & 1.13 & No signal \\
AuNPs (1L)+calix (2L) & 0.92 & 1.30 & 2.63 & 2.54 \\
\hline
\end{tabular}

exhibited the highest response to polar analytes. The ligand overlap regions between adjacent AuNPs are saturated hydrocarbon chains that allow non-polar molecules (i.e., benzene or toluene) to be partitioned within them and effectively cause film swelling. If a polar compound was to be absorbed in this film, it would be less favourable to partitioning within a non-polar region [40-42].

In direct contradiction with this result, investigations performed using SPR technique show the opposite behaviour. The DCM vapour has the highest effect on the hybrid film, while the minimum response was observed for benzene exposure. The interaction mechanism should not be explained with the same parameters for SPR technique where the SPR sensitivity depends on the optical parameters and the thickness of the thin films as already been explained by equation (2). It is very well known that the interaction of gas molecules with the thin film is believed to result in the swelling of the thin film because of the diffusion of the gas molecules into the bulk of the thin film. When the gas molecules diffuse into the bulk of the thin film the refractive index of the thin film may change because of the differences of the refractive indexes among the thin film and the gas molecules. When the refractive index of the analyte molecules is considered it is noteworthy that DCM with the lowest refractive index value $(n=1.42)$ among all analytes shows the highest response, whereas benzene with the highest refractive index value $(n=1.50)$ among all analytes exhibits the lowest response, keeping in mind that the refractive index of the thin film was deduced as 2.61 by the fitting of the SPR curves, which is not close to the analyte gases refractive index values.

\section{Conclusion}

Hybrid thin films of AuNPs-calixarene molecules as well as AuNPs monolayer and calixarene bilayer thin films have been deposited using LS techniques. The fabrication quality of the thin films has been checked using the UV-visible spectra and the SPR curve measurements of the thin films. The results obtained using both techniques are consistent with the literature. The thickness and optical parameters of hybrid thin film of the calixarene molecules fabricated onto the AuNPs monolayer have been reported for the first time. Gas sensing measurements were performed using UVvisible spectroscopy and surface plasmon resonance technique. The gas sensing results showed that the hybrid system revealed enhanced gas sensing behaviour for most of the studied analysts.

\section{References}

[1] Li H and Yang Y W 2013 Chin. Chem. Lett. 24545

[2] Cioffi N, Colaianni L, Ieva E, Pilolli R, Ditaranto N, Angione M D et al 2011 Electrochim. Acta 563713

[3] Gautam M and Jayatissa A H 2012 Solid-State Electron. 78 159

[4] Xia X, Guo S, Zhao W, Xu P, Yu H, Xu T and Li X 2014 Sens. Actuators B 202846

[5] Manera M G, Spadavecchia J, Buso D, de Julián Fernández C, Mattei G, Martucci A et al 2008 Sens. Actuators B 132107

[6] Rai P, Kim Y S, Song H M, Song M K and Yu Y T 2012 Sens. Actuators B $\mathbf{1 6 5} 133$

[7] Zhang J, Liu X, Wu S, Cao B and Zheng S 2012 Sens. Actuators B $\mathbf{1 6 9} 61$

[8] Cioffi N, Farella I, Torsi L, Valentini A and Tafuri A 2002 Sens. Actuators B $\mathbf{8 4} 49$

[9] Leng B, Zou L, Jiang J and Tian H 2009 Sens. Actuators B 140162

[10] Ozmen M, Ozbek Z, Bayrakci M, Ertul S, Ersoz M and Capan R 2014 Sens. Actuators B 195156

[11] Ozmen M, Ozbek Z, Buyukcelebi S, Bayrakci M, Ertul S, Ersoz M and Capan R 2014 Sens. Actuators B 190502

[12] Nabok A V, Hassan A K, Ray A K, Omar O and Kalchenko V I 1997 Sens. Actuators B 45115

[13] Özbek Z, Çapan R, Göktas H, Şen S, İnce F G, Özel M E and Davis F 2011 Sens. Actuators B 158235

[14] Ohira S I, Wanigasekara E, Rudkevich D M and Dasgupta P K 2009 Talanta 771814

[15] Richardson T H, Brook R A, Davis F and Hunter C A 2006 Colloids Surf. A 284-285 320

[16] Wang C, He X W and Chen L X 2002 Talanta 571181

[17] Liu C J, Lin J T, Wang S H, Jiang J C and Lin L G 2005 Sens. Actuators B 108521

[18] Sayin S, Ozbek C, Okur S and Yilmaz M 2014 J. Organomet. Chem. 7719

[19] Sun Y, Yao Y, Yan C G, Han Y and Shen M 2010 ACS Nano 42129

[20] Liang Q, Li C, Chen G and Jiang M 2012 J. Colloid Interface Sci. 38382

[21] Maity D, Gupta R, Gunupuru R, Srivastava D N and Paul P 2014 Sens. Actuators B 191757

[22] Maity D, Kumar A, Gunupuru R and Paul P 2014 Colloids Surf. A $\mathbf{4 5 5} 122$

[23] Usha S P, Mishra S K and Gupta B D 2015 Materials 82204

[24] Yang D 2011 Advances in nanocomposites - synthesis, characterization and industrial applications (Ch. 37, InTech)

[25] http://wwww.plasmachem.com

[26] Abbas R R, Richardson T H, Hobson A, Hassan A K and Abbas T R 2014 Colloids Surf. A 44495

[27] Ocak Ü, Ocak M and Bartsch R A 2012 Inorg. Chim. Acta 38144

[28] Ozbek Z, Erdogan M and Capan R 2014 Sens. Actuators B 196328

[29] Hardy N J and Richardson T H 2008 Colloids Surf. A 321285 
[30] Heriot S Y, Li Zhang H, Evans S D and Richardson T H 2006 Colloids Surf. A $\mathbf{2 7 8} 98$

[31] Knoll W 1998 Ann. Rev. Phys. Chem. 49569

[32] Hassan A K, Goy C and Nabok A V 2008 Thin Solid Films 5169006

[33] Chen M and Horn R G 2007 J. Colloid Interface Sci. 315814

[34] McCartney C M, Cowlam N, Davis F, Richardson T, Desert A, Gibaud A and Stirling C J M 2013 Colloids Surf. A 43641

[35] Pisarenko A N, Spendel W U, Taylor R T, Brown J D, Cox J A and Pacey G E 2009 Talanta 80777

[36] Capan I, Erdogan M, Stanciu G A, Stanciu S G, Hristu R and Göktepe M 2012 Mater. Chem. Phys. 1361130
[37] Acikbas Y, Capan R, Erdogan M and Yukruk F 2015 Appl. Surf. Sci. 350135

[38] Wen C, Yea Q, Zhang S and Wu D 2016 Sens. Actuators B 223791

[39] Basova T V, Mikhaleva N S, Hassan A K and Kiselev V G 2016 Sens. Actuators B 227634

[40] Im J, Sengupta S K, Baruch M F, Granz C D, Ammu S, Manohar S K and Whitten J E 2011 Sens. Actuators B 156715

[41] Jian R S, Huang R X and Lu C J 2012 Talanta 88160

[42] Kim Y J, Yang Y S, Ha S C, Cho S M, Kim Y S, Kim H Y, Yang H and Kim Y T 2005 Sens. Actuators B 106189 